\title{
Robust model predictive control of constrained linear systems with bounded disturbances ${ }^{1}$
}

\author{
D. Q. Mayne ${ }^{\mathrm{a}}$, M. M. Seron ${ }^{\mathrm{b}}$ and S. V. Raković ${ }^{\mathrm{a}}$ \\ ${ }^{a}$ Department of Electrical and Electronic Engineering, Imperial College London. \\ ${ }^{\mathrm{b}}$ School of Electrical Engineering and Computer Science, University of Newcastle, New South Wales, Australia.
}

\begin{abstract}
This paper provides a novel solution to the problem of robust model predictive control of constrained, linear, discrete-time systems in the presence of bounded disturbances. The optimal control problem that is solved online includes, uniquely, the initial state of the model employed in the problem as a decision variable. The associated value function is zero in a disturbance invariant set that serves as the 'origin' when bounded disturbances are present, and permits a strong stability result, namely robust exponential stability of the disturbance invariant set for the controlled system with bounded disturbances, to be obtained. The resultant online algorithm is a quadratic program of similar complexity to that required in conventional model predictive control.
\end{abstract}

Key words: Robust model predictive control, robustness, bounded disturbances

\section{Introduction}

Model predictive control is widely employed for the control of constrained systems and an extensive literature on the subject exists some of which is reviewed in [13]. Several methods for achieving robustness have been considered. The simplest is to ignore the disturbance and rely on the inherent robustness of deterministic model predictive control applied to the nominal system $[4,5]$. Open-loop model predictive control that determines the current control action by solving on-line an optimal control problem in which the decision variable is a sequence $\left\{u_{0}, u_{1}, \ldots, u_{N-1}\right\}$ of control actions was proposed in [6]; this method cannot contain the 'spread' of predicted trajectories resulting from disturbances. Hence feedback model predictive control in which the decision variable is a policy $\pi$, which is a sequence $\left\{\mu_{0}(\cdot), \mu_{1}(\cdot), \ldots, \mu_{N-1}(\cdot)\right\}$ of control laws, was advocated in, for example, [7-13]. Determination of a control policy is usually prohibitively difficult so research has concentrated on various simplifying approximations $[7,8,10,14-21]$. In many papers, following [17], a suboptimal control policy in which $\mu_{i}(x)=v_{i}+K x$ is employed.

$\overline{1}$ Research supported by the Engineering and Physical Sciences Research Council, UK
An important problem that arises in robust model predictive control when the disturbances are merely bounded is that asymptotic stability of the origin cannot be established; the best that can be achieved is robust asymptotic stability of a set $Z$. It is then useful to have a Lyapunov function that is zero in this set (since the value of the function cannot be guaranteed to decrease in this set); the set $Z$ may be regarded as the 'origin' for the uncertain system. This requirement on the Lyapunov function has been addressed in various ways in the literature. In [10] a terminal cost that is zero and a stage cost that is zero in $Z$ and strictly positive outside was proposed; the value function for the resultant optimal control problem is zero in the set $Z$. A disadvantage of this method is the discontinuous nature of the stage cost. An improved proposal [22] uses a continuous stage cost and zero terminal cost but requires solution of a quadratic program to evaluate the stage cost. An alternative approach [17] is to use a stage cost $\left|v_{i}\right|^{2}$ (when the suboptimal policy $\pi$ in which $\mu_{i}(x)=v_{i}+K x$ is employed) and a terminal cost of zero. With this approach, the stage cost does not involve the state, which is restrictive; attractivity, but not Lyapunov stability, of the set $Z$ has been established.

In this paper we adopt a novel approach to obtain a suitable Lyapunov function that has value zero in the set $Z$. The novelty is due to the fact that, in the proposed proce- 
dure, the initial state of the nominal (or reference) model employed in the optimal control problem solved online is a decision variable in addition to the usual control sequence. The incorporation of the initial state yields several advantages: firstly, the value function is zero in $Z$, facilitating the proof of both robust attractivity and robust stability of $Z$; secondly, the effective terminal set for the 'tube' of trajectories emanating from the initial state in the uncertain case is not restricted, as in prior literature, to be $Z$ which is analogous to a terminal set $\{0\}$ in the deterministic case, a restrictive requirement; thirdly it is possible to establish that the set $Z$ is robustly exponentially stable for the controlled system.

Some preliminary results are presented in $\S 2$ and $\S 3$. The new model predictive controller for bounded disturbances is presented in $\S 4$ where robust exponential stability of the set $Z$ is proved. Some conclusions are drawn in $\S 5$. An extension of these results to the case when the disturbance is exponentially decaying can be found in [23].

Notation: Given two sets $A, B$, then $A \oplus B \triangleq\{a+b \mid$ $a \in A, b \in B\}$ (set addition) and $A \ominus B \triangleq\{a \mid$ $a \oplus B \subseteq A\}$ (set subtraction). A set $Z$ is disturbance (or robustly positive) invariant for the discrete-time system $x^{+}=f(x, w)$ where $w \in W$ if $f(x, w) \in Z$ for all $x \in Z, w \in W$. The distance of a point $z$ from a set $X$ is denoted by $d(z, X) \triangleq \inf \{|z-x| \mid x \in X\}$. The origin is exponentially stable (Lyapunov stable and exponentially attractive) for $x^{+}=f(x)$ with a region of attraction $X_{N}$ if there exists a $c>0$ and a $\gamma \in(0,1)$ such that any solution $x(\cdot)$ of $x^{+}=f(x)$ with initial state $x(0) \in X_{N}$ satisfies $|x(i)| \leq c \gamma^{i}|x(0)|$ for all $i \geq 0$. A set $Z$ is robustly exponentially stable for $x^{+}=f(x, w)$, $w \in W$, with a region of attraction $X_{N}$ if there exists a $c>0$ and a $\gamma \in(0,1)$ such that any solution $x(\cdot)$ of $x^{+}=f(x, w)$ with initial state $x(0) \in X_{N}$, and admissible disturbance sequence $w(\cdot)(w(i) \in W$ for all $i \geq 0)$ satisfies $d(x(i), Z) \leq c \gamma^{i} d(x(0), Z)$ for all $i \geq 0$.

\section{The uncertain system}

The discrete-time system to be controlled is described by

$$
x^{+}=A x+B u+w
$$

where $x \in \mathbb{R}^{n}$ is the state, $u \in \mathbb{R}^{m}$ the control, and $w \in \mathbb{R}^{n}$ is a bounded disturbance; $x^{+}$denotes the successor state. The system is subject to state and control constraints

$$
u \in \mathbb{U}, \quad x \in \mathbb{X}
$$

where $\mathbb{U} \subset \mathbb{R}^{m}$ is compact, $\mathbb{X} \subset \mathbb{R}^{n}$ is closed, and each set contains the origin in its interior. The disturbance $w$ is assumed to be bounded, i.e.

$$
w \in W
$$

where $W$ is compact and contains the origin (but may not have an interior). Let $\mathbf{u}$ denote the control sequence $\{u(0), u(1), \ldots, u(N-1)\}$ and $\mathbf{w}$ the disturbance sequence $\{w(0), w(1), \ldots, w(N-1)\}$. The solution of (1) at time $i$ when the initial state is $x$ (at time 0), and the control and disturbance sequences are, respectively, $\mathbf{u}$ and $\mathbf{w}$ is $\phi(i ; x, \mathbf{u}, \mathbf{w})$. Let the nominal (reference) system corresponding to (1) be defined by

$$
x^{+}=A x+B u
$$

and let $\bar{\phi}(i ; x, \mathbf{u})$ denote the solution of (4) at time $i$ when the initial state is $x$ and the control sequence is $\mathbf{u}$.

Suppose $K \in \mathbb{R}^{m \times n}$ is such that $A_{K} \triangleq A+B K$ is stable. Let $Z$ be a disturbance invariant set for the controlled uncertain system $x^{+}=A_{K} x+w$ satisfying, therefore

$$
A_{K} Z \oplus W \subseteq Z .
$$

where $\oplus$ denotes Minkowski set addition. To reduce conservativeness, it is desirable that $Z$ be as small as possible. The minimal disturbance invariant set [24] is $\sum_{i=0}^{\infty} A_{K}^{i} W$ (with $\Sigma$ denoting here set addition) but this set is not necessarily polytopic unless controller $u=K x$ is deadbeat [25] or if $K$ is such that $A_{K}^{s}=\beta I$ for some finite integer $s$ and $\beta \in(0,1)$ [24]; however, a polytopic, disturbance invariant, outer approximation of the minimal disturbance set may be computed [26]. The set $Z$ serves as the 'origin' for the perturbed system. We require the following elementary result [18] which we use in $\S 4$ to define the new controller:

Proposition 1 Suppose $Z$ is disturbance invariant for $x^{+}=A_{K} x+w$. If $x \in \bar{x} \oplus Z$ and $u=\bar{u}+K(x-\bar{x})$, then $x^{+} \in \bar{x}^{+} \oplus Z$ for all $w \in W$ where $x^{+}=A x+B u+w$ and $\bar{x}^{+}=A \bar{x}+B \bar{u}$.

Proposition 1 states the feedback policy $u(i)=\bar{u}(i)+$ $K(x(i)-\bar{x}(i))$ keeps the states $x(i)$ of the uncertain system $x^{+}=A x+B u+w$ close to the states $\bar{x}(i)$ of the nominal system $\bar{x}^{+}=A \bar{x}+B \bar{u}$ (for all $\bar{u}(\cdot), x(i) \in$ $\bar{x}(i) \oplus Z$ if $x(0) \in \bar{x}(0) \oplus Z)$.

\section{Preliminary results}

The model predictive controller we propose uses the solution of an optimal control problem in which the initial state of the nominal (reference) model is a decision variable. The properties of the value function for this new problem are related to the properties of a conventional optimal control problem in which $\mathbf{u}$ is the sole decision variable. Hence, in this section we recall some relevant results for the conventional problem.

The conventional optimal control problem $\mathbb{P}_{N}(x)$, in which $x$ is the current state, has no uncertainties and has 
constraints that are tighter than the original (defined in (2)) precisely in order to guarantee that the final controller for the uncertain system ensures satisfaction of the original constraints. Problem $\mathbb{P}_{N}(x)$ is defined by:

$$
\begin{aligned}
& V_{N}^{0}(x)=\min _{\mathbf{u}}\left\{V_{N}(x, \mathbf{u}) \mid \mathbf{u} \in \mathcal{U}_{N}(x)\right\} \\
& \mathbf{u}^{0}(x)=\arg \min _{\mathbf{u}}\left\{V_{N}(x, \mathbf{u}) \mid \mathbf{u} \in \mathcal{U}_{N}(x)\right\}
\end{aligned}
$$

where the cost function $V_{N}(\cdot)$ is defined by

$$
V_{N}(x, \mathbf{u}) \triangleq \sum_{i=0}^{N-1} \ell(x(i), u(i))+V_{f}(x(N))
$$

For each $i, x(i)=\bar{\phi}(i ; x, \mathbf{u})$, and $\mathcal{U}_{N}(x)$ is the set of control sequences satisfying the tighter control, state and terminal constraints defined by

$$
\begin{aligned}
u(i) & \in \overline{\mathbb{U}} \triangleq \mathbb{U} \ominus K Z, i \in \mathcal{I}_{N-1} \\
x(i) & \in \overline{\mathbb{X}} \triangleq \mathbb{X} \ominus Z, i \in \mathcal{I}_{N-1} \\
x(N) & \in X_{f} \subset \mathbb{X} \ominus Z
\end{aligned}
$$

where $\mathcal{I}_{N-1} \triangleq\{0,1,2, \ldots, N-1\}$, and $X_{f}$ is the terminal constraint set for $\mathbb{P}_{N}(x) ; X_{f}$ is assumed to have an interior. As before, $x(i)$ denotes $\bar{\phi}(i ; x, \mathbf{u})$. Hence

$$
\begin{array}{r}
\mathcal{U}_{N}(x)=\left\{\mathbf{u} \mid u(i) \in \overline{\mathbb{U}}, \bar{\phi}(i ; x, \mathbf{u}) \in \overline{\mathbb{X}}, \forall i \in \mathcal{I}_{N-1}\right. \\
\left.\bar{\phi}(N ; x, \mathbf{u}) \in X_{f}\right\}
\end{array}
$$

The domain of the value function $V_{N}^{0}(\cdot)$ is $\bar{X}_{N}$ defined by:

$$
\bar{X}_{N} \triangleq\left\{x \mid \mathcal{U}_{N}(x) \neq \emptyset\right\} .
$$

It is assumed that $W$ is small enough to ensure that $Z \subset$ interior $(\mathbb{X})$ and $K Z \subset$ interior $(\mathbb{U})$ (the minimal $Z$ is proportional to $W$ ). It is also assumed (in (8)) that

$$
\ell(x, u) \triangleq(1 / 2)\left[x^{\prime} Q x+u^{\prime} R u\right], V_{f}(x) \triangleq(1 / 2) x^{\prime} P x
$$

where $Q, R$ and $P$ are positive definite, and that the terminal cost $V_{f}(\cdot)$ and the terminal constraint set $X_{f}$ satisfy the usual axioms ([3], page 797), namely:

A1: $A_{K} X_{f} \subset X_{f}, X_{f} \subset \mathbb{X} \ominus Z, K X_{f} \subset \mathbb{U} \ominus K Z$, A2: $V_{f}\left(A_{K} x\right)+\ell(x, K x) \leq V_{f}(x) \forall x \in X_{f}$.

The solution of $\mathbb{P}_{N}(x)$ yields the optimal control sequence $\mathbf{u}^{0}(x) \triangleq\left\{u_{0}^{0}(x), u_{1}^{0}(x), \ldots, u_{N-1}^{0}(x)\right\}$ and the associated optimal state sequence $\mathbf{x}^{0}(x) \triangleq\left\{x_{0}^{0}(x), x_{1}^{0}(x)\right.$, $\left.\ldots, x_{N}^{0}(x)\right\}, x_{0}^{0}(x) \triangleq x$, where, for each $i, x_{i}^{0}(x) \triangleq$ $\bar{\phi}\left(i ; x, \mathbf{u}^{0}(x)\right)$. The (implicit) model predictive control law is, therefore,

$$
\kappa_{N}^{0}(x) \triangleq u_{0}^{0}(x)
$$

so that the nominal system, under model predictive control, satisfies

$$
x^{+}=A x+B \kappa_{N}^{0}(x)
$$

This control law ensures satisfaction (by the nominal system) of the tighter constraints for all initial states in $\bar{X}_{N}$. It also stabilizes the nominal system; this follows from the fact that, under the assumptions made above, the value function satisfies:

$$
V_{N}^{0}\left(A x+B \kappa_{N}^{0}(x)\right) \leq V_{N}^{0}(x)-\ell\left(x, \kappa_{N}^{0}(x)\right)
$$

as shown in [3]. It follows from (14), (17) and A1-A2, as shown in [3], that there exist constants $c_{2}>c_{1}>0$ such that, with $x^{+}=A x+B \kappa_{N}^{0}(x)$,

$$
\begin{aligned}
c_{1}|x|^{2} & \leq V_{N}^{0}(x), \forall x \in \bar{X}_{N} \\
V_{N}^{0}\left(x^{+}\right) & \leq V_{N}^{0}(x)-c_{1}|x|^{2}, \forall x \in \bar{X}_{N} \\
V_{N}^{0}(x) & \leq c_{2}|x|^{2}, \forall x \in X_{f}
\end{aligned}
$$

If $\bar{X}_{N}$ is bounded, which we assume, the origin is exponentially stable for the controlled nominal system $x^{+}=$ $A x+B \kappa_{N}^{0}(x)$ with a region of attraction $\bar{X}_{N}$. It is these properties that we exploit in the derivation of a new robust controller.

\section{Robust model predictive controller}

Given any $x \in \bar{X}_{N} \backslash Z$, it is not necessarily true that $V_{N}^{0}\left(A x+B \kappa_{N}^{0}(x)+w\right)$ is smaller than $V_{N}^{0}(x)$ for all $w \in W$. Hence, to establish robust asymptotic stability of $Z$, we propose a novel optimization problem that is solved online; the new optimization problem involves, uniquely, the initial state. This is permissible because the initial state $x_{0}$ in the optimal control problem is now not the current state $x$ of the plant, which cannot be instantaneously changed, but a parameter of the control law. The new optimal control problem $\mathbb{P}_{N}^{*}(x)$, solved online, is a modification of $\mathbb{P}_{N}(x)$ defined in $\S 3$; the new problem problem $\mathbb{P}_{N}^{*}(x)$ is defined by:

$$
\begin{gathered}
V_{N}^{*}(x)=\min _{x_{0}, \mathbf{u}}\left\{V_{N}\left(x_{0}, \mathbf{u}\right) \mid \mathbf{u} \in \mathcal{U}_{N}\left(x_{0}\right), x \in x_{0} \oplus Z\right\} \\
\left(x_{0}^{*}(x), \mathbf{u}^{*}(x)\right)=\arg \min _{x_{0}, \mathbf{u}}\left\{V_{N}\left(x_{0}, \mathbf{u}\right) \mid \mathbf{u} \in \mathcal{U}_{N}\left(x_{0}\right),\right. \\
\left.x \in x_{0} \oplus Z\right\}
\end{gathered}
$$

In problem $\mathbb{P}_{N}^{*}(x)$, the function $V_{N}(x, \mathbf{u})$ and the constraint set $\mathcal{U}_{N}(x)$ are defined as in $\S 3$; the only difference between $\mathbb{P}_{N}(x)$ and $\mathbb{P}_{N}^{*}(x)$ is that the initial state $x_{0}$ of the model employed in the latter problem is a decision variable that can be varied provided it satisfies the constraint

$$
x \in x_{0} \oplus Z \text {. }
$$

where $x$ is the state of the system being controlled. It is easily shown that $\mathbb{P}_{N}^{*}(x)$, like $\mathbb{P}_{N}(x)$, is a quadratic 
program. The solution of $\mathbb{P}_{N}^{*}(x)$ yields the optimal control sequence $\mathbf{u}^{*}(x) \triangleq\left\{u_{0}^{*}(x), u_{1}^{*}(x), \ldots, u_{N-1}^{*}(x)\right\}$ and the associated optimal state sequence $\mathbf{x}^{*}(x) \triangleq$ $\left\{x_{0}^{*}(x), x_{1}^{*}(x), \ldots, x_{N}^{*}(x)\right\}$ where, for each $i>0$, $x_{i}^{*}(x) \triangleq \bar{\phi}\left(i ; x_{0}^{*}(x), \mathbf{u}^{*}(x)\right)$ (the optimal initial state $x_{0}^{*}(x)$ is not necessarily equal to the current state $x$ of the system being controlled as it is in $\S 3)$. A pair $\left(x_{0}, \mathbf{u}\right)$ is feasible for $\mathbb{P}_{N}^{*}(x)$ if $x \in x_{0} \oplus Z$ and $\mathbf{u} \in \mathcal{U}_{N}\left(x_{0}\right)$ (i.e. u satisfies (9)-(11)). Motivated by the discussion following Proposition 1, we propose that control $u_{0}^{*}(x)+K\left(x-x_{0}^{*}(x)\right)$ be applied to the system if its current state is $x$. The implicit model predictive control law $\kappa_{N}^{*}(\cdot)$, yielded by the solution of $\mathbb{P}_{N}^{*}(x)$, is, therefore

$$
\kappa_{N}^{*}(x) \triangleq u_{0}^{*}(x)+K\left(x-x_{0}^{*}(x)\right) .
$$

The control applied to the system when its state is $x$ is $\kappa_{N}^{*}(x)$ and is not necessarily equal to $u_{0}^{*}(x)$, the first element in the sequence $\mathbf{u}^{*}(x)$, as in conventional model predictive control. Let $x$ be an arbitrary state in

$$
X_{N} \triangleq\left\{x \mid \exists x_{0} \text { such that } x \in x_{0} \oplus Z, \mathcal{U}_{N}\left(x_{0}\right) \neq \emptyset\right\},
$$

the domain of the value function $V_{N}^{*}(\cdot)$. The optimal control and state trajectories $\mathbf{u}^{*}(x)$ and $\mathbf{x}^{*}(x)$ (for problem $\left.\mathbb{P}_{N}^{*}(x)\right)$ satisfy $u_{i}^{*}(x) \in \mathbb{U} \ominus K Z$ and $x_{i}^{*}(x) \in \mathbb{X} \ominus Z$ for all $i \in \mathcal{I}_{N-1}$; in addition, $x_{N}^{*}(x) \in X_{f} \subset \mathbb{X} \ominus Z$. An important consequence of the definition of the optimal control problem $\mathbb{P}_{N}^{*}(x)$ is the following result:

Proposition 2 (i) The domain $X_{N}$ of $V_{N}^{*}(\cdot)$ satisfies $X_{N}=\bar{X}_{N} \oplus Z \subseteq \mathbb{X}$, where $\bar{X}_{N}$ is the domain of $V_{N}^{0}(\cdot)$. (ii) $V_{N}^{*}(x)=\bar{V}_{N}^{0}\left(x_{0}^{*}(x)\right)$ and $\mathbf{u}^{*}(x)=\mathbf{u}^{0}\left(x_{0}^{*}(x)\right)$ for all $x \in X_{N}$. (iii) For all $x \in Z, V_{N}^{*}(x)=0$, $x_{0}^{*}(x)=0, \mathbf{u}^{*}(x)=\{0,0, \ldots, 0\}, \mathbf{x}^{*}(x)=\{0,0, \ldots, 0\}$ and $\kappa_{N}^{*}(x)=K x$.

Proof: (i) It follows from the definitions of $X_{N}$ and $\bar{X}_{N}$ that $X_{N}=\left\{x \mid \exists x_{0} \in \bar{X}_{N}\right.$ such that $\left.x \in x_{0} \oplus Z\right\}$ $=\bar{X}_{N} \oplus Z$. (ii) Follows from the definitions of $V_{N}^{*}(\cdot)$ and $V_{N}^{0}(\cdot)$. (iii) Let $x$ be an arbitrary point in $Z$. Since $x \in 0 \oplus Z$, it follows from the definition of $\mathbb{P}_{N}^{*}(x)$ that the pair $(0, \mathbf{0})$ (where $\mathbf{0}$ is the zero control sequence $\{0,0, \ldots 0\})$ is feasible for $\mathbb{P}_{N}^{*}(x)$. Hence $V_{N}^{*}(x) \leq V_{N}(0, \mathbf{0})=0$ which establishes the desired result.

The next result shows that the model predictive control law $\kappa_{N}^{*}(\cdot)$, unlike the nominal control law, $\kappa_{N}^{0}(\cdot)$, reduces the Lyapunov function at all $x \in X_{N}$ despite the additive bounded disturbance $w$.

Proposition 3 Suppose $x \in X_{N}$ so that $\left(x_{0}^{*}(x), \mathbf{u}^{*}(x)\right)$, where $\mathbf{u}^{*}(x)=\left\{u_{0}^{*}(x), u_{1}^{*}(x), \ldots, u_{N-1}^{*}(x)\right\}$, exists and is feasible for $\mathbb{P}_{N}^{*}(x) ; \mathbf{x}^{*}(x)=\left\{x_{0}^{*}(x), x_{1}^{*}(x), \ldots, x_{N}^{*}(x)\right\}$ is the associated state trajectory (for the nominal system). Then, for all $x^{+} \in A x+B \kappa_{N}^{*}(x) \oplus W,\left(x_{1}^{*}(x), \tilde{\mathbf{u}}(x)\right)$ with $\tilde{\mathbf{u}}(x)$ defined by

$$
\tilde{\mathbf{u}}(x) \triangleq\left\{u_{1}^{*}(x), u_{2}^{*}(x), \ldots, u_{N-1}^{*}(x), K x_{N}^{*}(x)\right\},
$$

is feasible for $\mathbb{P}_{N}^{*}\left(x^{+}\right)$, and

$$
V_{N}^{*}\left(x^{+}\right) \leq V_{N}^{*}(x)-\ell\left(x_{0}^{*}(x), \kappa_{N}^{0}\left(x_{0}^{*}(x)\right)\right) .
$$

Proof: The state sequences associated with $\mathbf{u}^{*}(x)$ and $\tilde{\mathbf{u}}(x)$ are, respectively, $\mathbf{x}^{*}(x)$ and $\tilde{\mathbf{x}}(x)$ where

$$
\tilde{\mathbf{x}}(x)=\left\{x_{1}^{*}(x), x_{2}^{*}(x), \ldots, x_{N}^{*}(x), A_{K} x_{N}^{*}(x)\right\}
$$

Because $x \in x_{0}^{*}(x) \oplus Z$, it follows, from Proposition 1 and (23), that $x^{+} \in x_{1}^{*}(x) \oplus Z$. Since $\left(x^{*}(x), \mathbf{u}^{*}(x)\right)$ is feasible for $\mathbb{P}_{N}^{*}(x)$, constraints (9)-(11) are satisfied by $\mathbf{u}^{*}(x)$ and $\mathbf{x}^{*}(x)$. Hence constraint (9) is satisfied by the first $N-1$ elements of $\tilde{\mathbf{u}}(x)$ and constraint (10) is satisfied by the first $N$ elements of $\tilde{\mathbf{x}}(x)$. Since $x_{N}^{*}(x) \in X_{f}$, it follows from $\mathbf{A} 1$ that $K x_{N}^{*}(x) \in \mathbb{U} \ominus K Z$ and $A_{K} x_{N}^{*}(x) \in X_{f}$. Hence the last element $K x_{N}^{*}(x)$ of $\tilde{\mathbf{u}}(x)$ satisfies constraint (9) and the last element $A_{K} x_{N}^{*}(x)$ of $\tilde{\mathbf{x}}(x)$ satisfies constraint (11). Thus $\tilde{\mathbf{u}}(x) \in \mathcal{U}_{N}\left(x_{1}^{*}(x)\right)$. Since $x^{+} \in$ $x_{1}^{*}(x) \oplus Z$ and $\tilde{\mathbf{u}}(x) \in \mathcal{U}_{N}\left(x_{1}^{*}(x)\right)$, the pair $\left(x_{1}^{*}(x), \tilde{\mathbf{u}}(x)\right)$ is feasible for $\mathbb{P}_{N}^{*}\left(x^{+}\right)$and $x^{+} \in X_{N}$. To prove (24), note that $x^{+} \in x_{1}^{*}(x) \oplus Z$ so that $\left(x_{1}^{*}(x), \mathbf{u}^{0}\left(x_{1}^{*}(x)\right)\right)$ is feasible for $\mathbb{P}_{N}^{*}\left(x^{+}\right)$. Hence $V_{N}^{*}\left(x^{+}\right) \leq V_{N}^{0}\left(x_{1}^{*}(x)\right)$. But, from $(17), V_{N}^{0}\left(x_{1}^{*}(x)\right) \leq V_{N}^{0}\left(x_{0}^{*}(x)\right)-\bar{\ell}\left(x_{0}^{*}(x), \kappa_{N}^{0}\left(x_{0}^{*}(x)\right)\right.$ since $x_{1}^{*}(x)$ is the state of the nominal system at time 1 if at time 0 its state is $x_{0}^{*}(x)$ and its control is $\kappa_{N}^{0}\left(x_{0}^{*}(x)\right)=$ $u_{0}^{0}\left(x_{0}^{*}(x)\right)$; since $V_{N}^{*}(x)=V_{N}^{0}\left(x_{0}^{*}(x)\right)$, (24) follows.

The main result of this section follows.

Theorem 1 The set $Z$ is robustly exponentially stable for the controlled uncertain system $x^{+}=A x+B \kappa_{N}^{*}(x)+$ $w$ where $w \in W$. The region of attraction is $X_{N}$.

Proof: From Proposition 2, $V_{N}^{*}(x)=V_{N}^{0}\left(x_{0}^{*}(x)\right)$ for all $x \in X_{N}$. Hence, using (18)-(20) and (24), we obtain:

$$
\begin{aligned}
& V_{N}^{*}(x)=V_{N}^{0}\left(x_{0}^{*}(x)\right) \geq c_{1}\left|x_{0}^{*}(x)\right|^{2}, \forall x \in X_{N}, \\
& V_{N}^{*}\left(x^{+}\right)-V_{N}^{*}(x) \leq-\ell\left(x_{0}^{*}(x), \kappa_{N}^{0}\left(x_{0}^{*}(x)\right)\right) \leq \\
& -c_{1}\left|x_{0}^{*}(x)\right|^{2}, \forall x \in X_{N}, \forall x^{+} \in\left(A x+B \kappa_{N}^{*}(x)\right) \oplus W \\
& V_{N}^{*}(x)=V_{N}^{0}\left(x_{0}^{*}(x)\right) \leq c_{2}\left|x_{0}^{*}(x)\right|^{2}, \forall x \in X_{f} \oplus Z .
\end{aligned}
$$

where $c_{2}>c_{1}$. Let $x(i)$ denote the solution of $x^{+}=$ $A x+B \kappa_{N}^{*}(x)+w$ given its initial state is $x(0)=x$ and disturbance is $w(\cdot)$; from Proposition $3, x(i) \in X_{N}$ for all $i$. For all $\alpha \geq 0$, let $S_{\alpha} \triangleq\left\{x \mid V_{N}^{*}(x) \leq \alpha\right\}$. Then $S_{0}=Z$ and there exists an $\alpha>0$ such that $S_{\alpha} \subset X_{f} \oplus Z$. From $(25)-(27), V_{N}^{*}(x(i)) \leq \gamma^{i} V_{N}^{*}(x(0)), \gamma \triangleq\left(1-c_{1} / c_{2}\right) \in$ 
$(0,1)$ and $\left|x_{0}^{*}(x(i))\right| \leq c \delta^{i}\left|x_{0}^{*}(x(0))\right|, \delta \triangleq \sqrt{\gamma}$, for all $x(0)=x \in S_{\alpha}$, some $c<\infty$. Since $\bar{X}_{N}$ and, hence, $X_{N}$ are bounded, it follows from (26) that there exists a finite integer $I$ such that $x(i) \in S_{\alpha}$ for all $i \geq I$, all $x \in X_{N}$, and every admissible disturbance $w(\cdot)$. Hence there exists a $d>c$ such that $\left|x_{0}^{*}(x(i))\right| \leq d \delta^{i}\left|x_{0}^{*}(x(0))\right|$ for all $x(0)=x \in X_{N}$ and every admissible disturbance $w(\cdot)$. Since $x(i) \in x_{0}^{*}(x(i)) \oplus Z$, the set $Z$ is robustly exponentially stable for the controlled uncertain system with a region of attraction $X_{N}$.

\subsection{Illustrative example}

The numerical example is control of the constrained, sampled double integator defined by:

$$
x^{+}=\left[\begin{array}{ll}
1 & 1 \\
0 & 1
\end{array}\right] x+\left[\begin{array}{c}
0.5 \\
1
\end{array}\right] u+w
$$

The state constraints are $x \in \mathbb{X} \triangleq\left\{x \mid\left[\begin{array}{ll}0 & 1\end{array}\right] x \leq 2\right\}$; the control constraint is $u \in \mathbb{U} \triangleq\{u|| u \mid \leq 1\}$ and the disturbance is bounded: $w \in W \triangleq\left\{w \mid\|w\|_{\infty} \leq 0.1\right\}$. The cost function is defined by (8) and (14) with $Q=I$, $R=0.01$; the terminal cost $V_{f}(x)$ is the value function $(1 / 2) x^{\prime} P x$ for the optimal unconstrained problem for the nominal system so that

$$
P=\left[\begin{array}{ll}
2.0066 & 0.5099 \\
0.5099 & 1.2682
\end{array}\right]
$$

and $u=K x$ is the optimal unconstrained controller for $(A, B, Q, R)$. The set $Z$ is computed as a polytopic, disturbance invariant, outer invariant approximation of the minimal disturbance set [26] and the terminal constraint set $X_{f}$ satisfies A1. The horizon length is $N=9$. The sets $X_{9}$ and $\bar{X}_{9}$ (the domains of $V_{N}^{*}(\cdot)$ and $V_{N}^{0}(\cdot)$, respectively) are shown in Figure 1. In Figure 2, a state

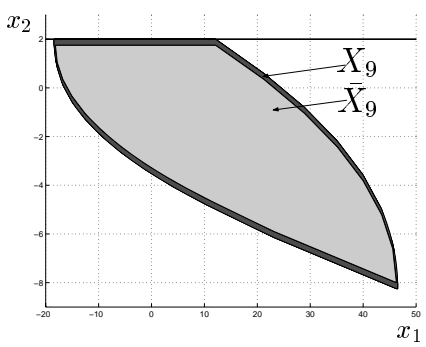

Fig. 1. Feasibility Sets for example of $\S 4.1$

trajectory for initial condition $x_{0}=(-5,-2)^{\prime}$ is shown; the dash-dot line is the actual trajectory $\{x(i)\}$ for a sequence of extreme disturbances while the solid line is the sequence $\left\{x_{0}^{*}(x(i))\right\}$ of optimal initial states. The sets $x_{0}^{*}(x(i)) \oplus Z$ are shown shaded in Figure 2. Also shown in Figure 2 are the sets $X_{f}$ and the set $X_{f} \oplus Z$ which is the effective terminal set for the 'tube' of trajectories; $X_{f} \oplus Z$ can be much larger than $Z$.

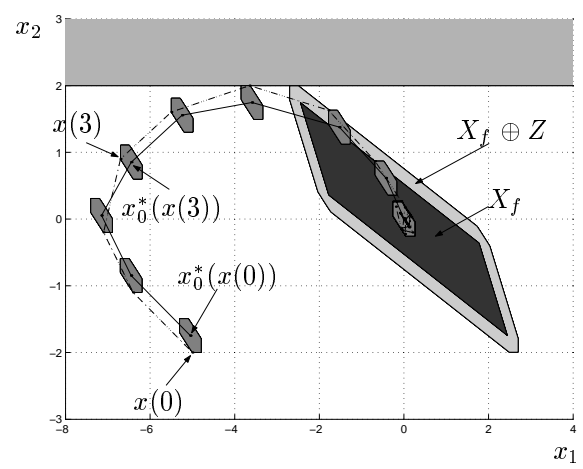

Fig. 2. Trajectories for example of $\S 4.1$

\section{Conclusion}

We have presented a robust model predictive controller for constrained linear systems with bounded disturbances. A novel feature of the robust model predictive controller is the fact that the decision variable in the (nominal) optimal control problem solved online incorporates the initial state of the model as well as the control sequence. The resultant value function is zero in the disturbance invariant set $Z$ permitting robust exponential stability of $Z$ to be established. The controller is relatively simple, merely requiring, in the optimal control problem solved online, optimization over the initial state $x_{0}$ of the model and a sequence of control actions subject to satisfaction of a tighter set of constraints than in the original problem; the optimal control problem is a standard quadratic program of approximately the same complexity as that required for conventional model predictive control (the dimension of the decision variable is increased by $n$ ). The results depend on linearity and cannot easily be extended to nonlinear systems.

\section{References}

[1] A. Bemporad and M. Morari. Robust model predictive control: a survey. In A. Garulli, A. Tesi, and A. Vicino, editors, Robustness in Identification and Control. SpringerVerlag, Boston, USA, 1999.

[2] Frank Allgöwer, Thomas A. Badgwell, Joe S. Qin, James B. Rawlings, and Stephen J. Wright. Nonlinear predictive control and moving horizon estimation - an introductory overview. In Paul M. Frank, editor, Advances in Control: highlights of ECC'99, pages 391-449, London, 1999. Springer.

[3] D. Q. Mayne, J. B. Rawlings, C. V. Rao, and P. O. M. Scokaert. Constrained model predictive control: stability and optimality. Automatica, 36:789-814, June 2000.

[4] P. O. M. Scokaert and J. B. Rawlings. Stability of model predictive control under perturbations. In Proceedings of the IFAC symposium on nonlinear control systems design, Lake Tahoe, California, June 1995.

[5] D. Limón Marruedo, T. Álamo, and E. F. Camacho. Stability analysis of systems with bounded additive uncertainties based on invariant sets: stability and feasibility of MPC. In Proceedings American Control Conference, pages 364-369, Anchorage, Alaska, May 2002. 
[6] A. Zheng and M. Morari. Robust stability of constrained model predictive control. In Proceedings of the 1993 American Control Conference, pages 379-383, 1993.

[7] D. Q. Mayne. Optimization in model based control. In Proceedings of the IFAC symposium on dynamics and control chemical reactors and batch processes (Dycord+'95), Helsingor, Denmark, pages 229-242. Elsevier Science, Oxford, 7-9 June 1995.

[8] M. V. Kothare, V. Balakrishnan, and M. Morari. Robust constrained model predictive control using linear matrix inequalities. Automatica, 32(10):1361-1379, 1996.

[9] J. H. Lee and Z. Yu. Worst-case formulations of model predictive control for systems with bounded parameters. Automatica, 33(5):763-781, 1997.

[10] P. O. M. Scokaert and D. Q. Mayne. Min-max feedback model predictive control for constrained linear systems. IEEE Transactions on Automatic Control, 43(8):1136-1142, August 1998.

[11] G. De Nicolao, L. Magni, and R. Scattolini. Stability and robustness of nonlinear model predictive control. In Frank Allgöwer and Alex Zheng, editors, Nonlinear Model Predictive Control, pages 3-22. Birkhäuser, Basle, 2000.

[12] L. Magni, H. Nijmeijer, and A. van der Schaft. A receding horizon approach to the nonlinear $H_{\infty}$ problem. Automatica, 37(3):429-435, 2001.

[13] L. Magni, G. De Nicolao, R. Scattolini, and F. Allgöwer. Robust model predictive control of nonlinear discrete-time systems. International Journal of Robust and Nonlinear Control, 13:229-246, 2003.

[14] B. G. Park and W. H. Kwon. Robust one-step receding horizon control for constrained systems. International Journal of Robust and Nonlinear Control, 9:381-395, 1999.

[15] B. Kouvaritakis, J. A. Rossiter, and J. Schuurmans. Efficient robust predictive control. IEEE Transactions on Automatic Control, 45(8):145-1549, 2000.

[16] J. Schuurmans and J. A. Rossiter. Robust model predictive control using tight sets of predicted states. Proceedings of the IEE, 147(1):13-18, January 2000.

[17] Young Il Lee and Basil Kouvaritakis. Robust receding horizon control for systems with uncertain dynamics and input saturation. Automatica, 36:1497-1504, 2000.

[18] D. Q. Mayne and W. Langson. Robustifying model predictive control of constrained linear systems. Electronics Letters, 37(23):1422-1423, 2001.

[19] L. Chisci, J. A. Rossiter, and G. Zappa. Systems with persistent disturbances: predictive control with restricted constraints. Automatica, 37:1019-1028, 2001.

[20] Y. I. Lee, B. Kouvaritakis, and M. Cannon. Constrained receding horizon predictive control for nonlinear systems. Automatica, 38(12), 2002.

[21] Wilbur Langson, Ioannis Chryssochoos, Sasa Rakovic̀, and David Mayne. Robust model predictive control using tubes. Automatica, 40:125-133, January 2004.

[22] Eric C. Kerrigan and Jan M. Maciejowski. Feedback minmax model predictive control using a single linear program: robust stability and the explicit solution. Technical Report CUED/F-INFENG/TR.440, Department of Engineering, University of Cambridge, 2003.

[23] D. Q. Mayne, M. Seron, and S. V. Raković. Robust model predictive control of constrained linear systems with bounded and exponentially decaying disturbances. Technical Report EEE/C\&P/DQM/11/2003, Department of Electrical and Electronic Engineering, Imperial College London, 2003.
[24] I. Kolmanovsky and E. C. Gilbert. Theory and computation of disturbance invariant sets for discrete-time linear systems. Mathematical Problems in Engineering, 4:317-367, 1998.

[25] D. Q. Mayne and W. R. Schroeder. Robust timeoptimal control of constrained linear systems. Automatica, 33(12):2103-2118, 1997.

[26] S. V. Raković, E. C. Kerrigan, K. I. Kouramas, and D. Q. Mayne. Invariant approximations of robustly positively invariant sets for constrained linear discrete-time systems subject to bounded disturbances. Technical Report CUED/F-INFENG/TR.473, Department of Engineering, University of Cambridge, UK, January 2004. Downloadable from http://www-control.eng.cam.ac.uk/eck21. 